\title{
On the Helly Number for Hyperplane Transversals to Unit Balls*
}

\author{
B. Aronov, ${ }^{1}$ J. E. Goodman, ${ }^{2}$ R. Pollack, ${ }^{3}$ and R. Wenger ${ }^{4}$ \\ ${ }^{1}$ Polytechnic University, \\ Brooklyn, NY 11201, USA \\ aronov@ziggy.poly.edu \\ ${ }^{2}$ City College, City University of New York, \\ New York, NY 10031, USA \\ jegcc@cunyvm.cuny.edu \\ ${ }^{3}$ Courant Institute of Mathematical Sciences, \\ New York University, \\ New York, NY 10012, USA \\ pollack@geometry.cims.nyu.edu \\ ${ }^{4}$ Ohio State University, \\ Columbus, OH 43210, USA \\ wenger@cis.ohio-state.edu
}

\begin{abstract}
We prove two results about the Hadwiger problem of finding the Helly number for line transversals of disjoint unit disks in the plane, and about its higher-dimensional generalization to hyperplane transversals of unit balls in $d$-dimensional Euclidean space. These consist of (a) a proof of the fact that the Helly number remains 5 even for arbitrarily large sets of disjoint unit disks - thus correcting a 40-year-old error; and (b) a lower bound of $d+3$ on the Helly number for hyperplane transversals to suitably separated families of unit balls in $\mathbb{R}^{d}$.
\end{abstract}

\section{Introduction}

In 1955 Hadwiger [12] posed the problem of determining the smallest number $k$ with the property that if every collection of $k$ members of a family of $n \geq k$ pairwise disjoint unit disks in the plane are met by a line, then all the disks are met by a line; i.e., the problem of determining the so-called Helly number (if one exists) for line transversals

* Boris Aronov was supported in part by a Sloan Research Fellowship, Jacob E. Goodman was supported in part by NSF Grant DMS-9322475, NSA Grant MDA904-98-I-0032, and PSC-CUNY Grant 668416, Richard Pollack was supported in part by NSF Grants CCR-9711240 and CCR-9732101 and NSA Grant MDA90498-I-0505, and Rephael Wenger was supported in part by NSA Grant MDA904-97-I-0019. 
to disjoint unit disks in the plane. He pointed out, by means of an example consisting of disks centered at the vertices of a regular pentagon and almost touching, that $k=4$ would not suffice. It is this problem, along with its generalization to higher dimensions, that we consider in this paper.

Hadwiger's problem was solved 2 years later in the planar case by Danzer [4], who gave an intricate case analysis to show that $k=5$ works.

In 1958, in a paper in which he extended Danzer's result to parallelograms, Grünbaum [10] asserted the following strengthened version of Danzer's theorem: "For families of disjoint, congruent circles containing at least six members, $T(4)$ implies $T$." (The notation $T(k)$, which has since come to be widely used, means that every $k$ members of the family have a (line) transversal; similarly, " $T$ " means that all do.) He provided only an outline of the proof.

It turns out, however, that this assertion is incorrect: even for arbitrarily large families of unit disks, $T(5)$ is necessary to get $T$. We give an example in Section 2 below. (This corrects an error that has become embedded in the literature during the past 40 years; see [5], [6], [8], and [13], for example.)

As Grünbaum points out [11], this means that the situation is now much clearer than before, in the following sense: In [10] he had conjectured that $T(5) \Rightarrow T$ for any family of disjoint translates of a compact convex set in the plane. This was finally proven in 1989 by Tverberg [16], thus generalizing Danzer's theorem on unit disks. It appeared, however, that the circular disk was exceptional among all planar convex sets, in that $T$ (4) was sufficient for $T$ for its translates (in a family of sufficient size). One sees now that this is not the case after all.

In [4] Danzer also considered briefly two variants of the problem in higher dimensions: one for line transversals of pairwise disjoint unit balls (which we will not touch on here), the other for hyperplane transversals. In the latter case he observed that, in $\mathbb{R}^{3}$, no Helly number exists for pairwise disjoint unit balls.

In his example, however, the balls are permitted to have line transversals for more than just two balls; it is this that allows the result to fail, just as the existence of a Helly number for line transversals in the plane would fail if the disks were permitted to have points in common-see Section 4 of [4]. As demonstrated in a number of recent papers [2], [3], [7], [9], [14], [15], [17], the appropriate generalization of "pairwise disjoint," when considering $k$-dimensional transversals, is " $(k-1)$-separated": A family of convex sets in $\mathbb{R}^{d}$ is $(k-1)$-separated if no $k+1$ of them have a $(k-1)$-transversal; thus " 0 -separated" means pairwise disjoint, and in the case where one is interested in the existence of a hyperplane transversal, "( $d-2)$-separated" means that no $d$ of the sets have a transversal of dimension less than $d-1$. If one assumes that the balls in question are $(d-2)$-separated, Danzer's remark does not apply, and there is no a priori reason why a Helly number should not exist.

It is this problem, of finding the Helly number for separated unit balls (and, more generally, for separated translates of a single compact convex set), that we set out initially to investigate. While we were unable to find an upper bound for the Helly number, or even to prove that one exists, in dimension greater than $2,{ }^{1}$ we did discover a lower

\footnotetext{
${ }^{1}$ We were, however, able to find an alternate proof [1] of Danzer's theorem that the Helly number in the plane is 5, which we believe is more transparent than the original one, and which we hope may generalize to higher dimensions.
} 
bound. This is presented in Section 3. There, we first prove a theorem about the strict monotonicity (as a function of $d$ ) of the Helly number, if one exists, for hyperplane transversals of $(d-2)$-separated unit balls in $\mathbb{R}^{d}$. Starting with the example in Section 2, this then allows us to construct, for each $d \geq 2$, an example of $d+3$ unit balls in $\mathbb{R}^{d}$, no $d$ having a $(d-2)$-transversal, for which every $d+2$ have a hyperplane transversal, yet all $d+3$ do not. This shows, in particular, that the Helly number for hyperplane transversals of $(d-2)$-separated unit balls in $\mathbb{R}^{d}$, if one exists, must be at least $d+3$.

Finally, we conjecture that such a Helly number does indeed exist:

Conjecture 1. For each $d>2$, there is a $k(d)$ such that, for hyperplane transversals to families of $(d-2)$-separated unit balls in $\mathbb{R}^{d}, T(k(d)) \Rightarrow T$.

For background and additional information on Helly's theorem and geometric transversals, we refer the reader to the excellent survey [5] and to several surveys containing more recent results [6], [8], [18], [19].

\section{2. $T(4) \nRightarrow T$}

The following example shows that $T(4)$ does not suffice for $T$, even in the case of six or more disjoint unit disks.

Theorem 1. For each $n \geq 6$, there exist $n$ pairwise disjoint unit disks in $\mathbb{R}^{2}$ such that any four have a common transversal but some five do not.

Proof. We first describe the construction for $n=6$, and then indicate how to extend it to $n>6$.

Begin with six unit disks centered at (say) $(0,0),(3,0),(10, \pm 1),(12, \pm 1)$. Then translate the fifth and sixth up and down (resp.) a distance $\varepsilon$, for $\varepsilon$ suitably small $(0.001$ will do), and translate the third and fourth up and down (resp.) a distance $\varepsilon^{2}$. Figure 1 shows the new disks (with the size of $\varepsilon$ exaggerated). Notice that the disks are now pairwise disjoint.

One checks easily (by calculating the distances from various centers to the lines tangent to appropriate pairs of disks) that every four disks are met by a line; disks $\Delta_{3}, \Delta_{4}, \Delta_{5}, \Delta_{6}$, for example, are met by a vertical line. On the other hand, since a vertical line is the only common transversal to $\Delta_{3}, \Delta_{4}, \Delta_{5}, \Delta_{6}$, disks $\Delta_{1}, \Delta_{3}, \Delta_{4}, \Delta_{5}, \Delta_{6}$ have no common transversal.
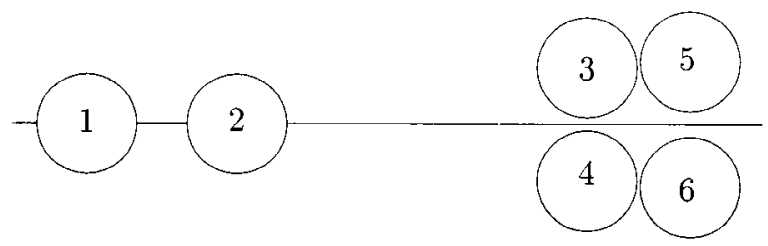

Fig. 1. Six unit disks. 
By making $\varepsilon$ successively smaller, we may add an arbitrarily large (finite) number of additional disks with centers at (say) $(-3,0),(-6,0), \ldots$, while maintaining $T(4)$ but avoiding $T$.

\section{A Lower Bound for the Hyperplane Transversal Helly Number in $\mathbb{R}^{d}$}

Danzer remarks in [4] that if $k_{d}$ is the smallest $k$ for which $T(k) \Rightarrow T$ for line transversals of disjoint unit balls in $\mathbb{R}^{d}$, then trivially $k_{d+1} \geq k_{d}$ for $d \geq 2$.

For hyperplane transversals to suitably separated unit balls, it turns out that the Helly number is, in fact, strictly monotone:

Theorem 2. If there is a collection of $n(d-2)$-separated unit balls in $\mathbb{R}^{d}$ for which $T(k)$ holds for hyperplanes, but not $T(k+1)$, then there is a collection of $n+1$ $(d-1)$-separated unit balls in $\mathbb{R}^{d+1}$ for which $T(k+1)$ holds for hyperplanes, but not $T(k+2)$.

Proof. Since the hypothesis remains valid if the given balls are enlarged very slightly, we may assume without loss of generality that for any $k$ of them there is a hyperplane cutting the interior of each.

Suppose the given balls, $\Gamma_{1}, \ldots, \Gamma_{n}$, have centers at $\left(x_{11}, \ldots, x_{1 d}\right), \ldots,\left(x_{n 1}, \ldots, x_{n d}\right)$, respectively. Embed $\mathbb{R}^{d}$ in $\mathbb{R}^{d+1}$ as the hyperplane of the first $d$ coordinates, and call it $H_{0}$. Let $\Delta_{1}, \ldots, \Delta_{n+1}$ be the unit balls in $\mathbb{R}^{d+1}$ centered at $\left(x_{11}, \ldots, x_{1 d}, M_{1}\right), \ldots,\left(x_{n 1}, \ldots\right.$, $\left.x_{n d}, M_{d}\right),\left(x_{n 1}, \ldots, x_{n d}, M_{d+1}\right)$, respectively, where the coordinates $M_{i}$ are chosen in the order $M_{1}, \ldots, M_{n+1}$, and each is chosen sufficiently larger than its predecessor, in a manner to be determined below; see Fig. 2. (Notice that the center of $\Gamma_{n}$ is used twice.)

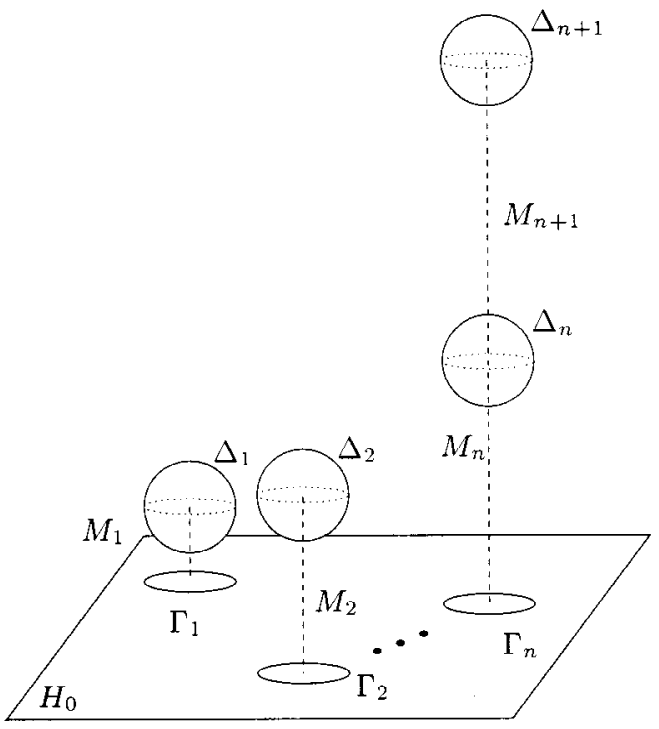

Fig. 2. The $n+1$ lifted balls. 
We must show that these $n+1$ balls satisfy all three conditions.

(i) They are $(d-1)$-separated. Suppose $\Delta_{i_{1}}, \ldots, \Delta_{i_{d+1}}$ are any $d+1$ of the balls, with $i_{1}<\cdots<i_{d+1}$. Since $\Gamma_{i_{1}}, \ldots, \Gamma_{i_{d}}$ have no $(d-2)$-transversal, a $(d-1)$-flat meeting $\Delta_{i_{1}}, \ldots, \Delta_{i_{d}}$ cannot be vertical. Hence if $M_{i_{d+1}}$ is chosen sufficiently large, the ball $\Delta_{i_{d+1}}$ will lie above all such hyperplanes. (If, for $M_{i_{d+1}}$ arbitrarily large, there were a hyperplane through $\Delta_{i_{1}}, \ldots, \Delta_{i_{d+1}}$, by compactness we could find a sequence of hyperplanes converging to a vertical one, each meeting $\Delta_{i_{1}}, \ldots, \Delta_{i_{d}}$; hence the limiting one would meet $\Delta_{i_{1}}, \ldots, \Delta_{i_{d}}$ as well, which is impossible.)

(ii) Any $k+1$ of the balls $\Delta_{1}, \ldots, \Delta_{n+1}$ have a hyperplane transversal. This is clear if both $\Delta_{n}$ and $\Delta_{n+1}$ are included among them: just take the vertical extension of a $(d-1)$ transversal to the $k$ distinct projections into the hyperplane $H_{0}$. If not, suppose the balls are $\Delta_{i_{1}}, \ldots, \Delta_{i_{k+1}}$ with $i_{1}<\cdots<i_{k+1}$. Let $F$ be a $(d-1)$-flat in the space $H_{0}$ cutting the interior of each of $\Gamma_{i_{1}}, \ldots, \Gamma_{i_{k}}$, and let $H$ be the hyperplane obtained by extending $F$ vertically. If $H$ is tilted by a sufficiently small amount, the resulting hyperplane $H^{\prime}$ will still meet all of the lifted balls $\Delta_{i_{1}}, \ldots, \Delta_{i_{k}}$; if $M_{i_{k+1}}$ is large enough, such an $H^{\prime}$ can be chosen to meet $\Delta_{i_{k+1}}$ as well.

(iii) Some $k+2$ of the balls $\Delta_{1}, \ldots, \Delta_{n+1}$ have no hyperplane transversal. Suppose $\Gamma_{i_{1}}, \ldots, \Gamma_{i_{k+1}}$ have no $(d-1)$-transversal in $H_{0}$. We claim that $\Delta_{i_{1}}, \ldots, \Delta_{i_{k+1}}, \Delta_{n+1}$ will have no $d$-transversal in $\mathbb{R}^{d+1}$ if $M_{n+1}$ is chosen large enough.

If, for each choice of $M_{n+1}$, no matter how large, we can find a hyperplane through $\Delta_{n+1}$ meeting all of $\Delta_{i_{1}}, \ldots, \Delta_{i_{k+1}}$, then (by the compactness of the space of hyperplanes meeting a ball containing all of $\Gamma_{1}, \ldots, \Gamma_{n}$ ) there is a sequence $H_{1}, H_{2}, \ldots$ of hyperplanes converging to a vertical hyperplane $H$, such that each $H_{i}$ meets all of $\Delta_{i_{1}}, \ldots, \Delta_{i_{k+1}}$. It follows that $H$ does as well, so that $H \cap H_{0}$ is a $(d-1)$-flat in $H_{0}$ meeting all of $\Gamma_{i_{1}}, \ldots, \Gamma_{i_{k+1}}$, contrary to hypothesis. Hence for $M_{n+1}$ large enough, $\Delta_{i_{1}}, \ldots, \Delta_{i_{k+1}}, \Delta_{n+1}$ will have no $d$-transversal.

Corollary 1. The Helly number for hyperplane transversals to families of $d+3$ or more $(d-2)$-separated unit balls in $\mathbb{R}^{d}$ is at least $d+3$.

Proof. This is immediate from Theorems 1 and 2, plus Hadwiger's example in [12].

\section{References}

1. B. Aronov, J. E. Goodman, R. Pollack, and R. Wenger, A new proof of Danzer's theorem on the Helly number for line transversals to unit disks. Manuscript.

2. T. Bisztriczky, On separated families of convex bodies. Arch. Math. 54 (1990), 193-199.

3. S. Cappell, J. E. Goodman, J. Pach, R. Pollack, M. Sharir, and R. Wenger, Common tangents and common transversals. Adv. in Math. 106 (1994), 198-215.

4. L. Danzer, Über ein Problem aus der kombinatorischen Geometrie. Arch. Math. (Basel) 8 (1957), 347-351.

5. L. Danzer, B. Grünbaum, and V. Klee, Helly's theorem and its relatives. In V. Klee, ed., Convexity, pp. 101180. Proceedings of Symposia in Pure Mathematics, 7. American Mathematical Society, Providence, RI, 1963.

6. J. Eckhoff, Helly, Radon, and Carathéodory type theorems. In P. M. Gruber and J. M. Wills, eds., Handbook of Convex Geometry, pp. 389-448. North-Holland, Amsterdam, 1993.

7. J. E. Goodman and R. Pollack, Hadwiger's transversal theorem in higher dimensions. J. Amer. Math. Soc. 1 (1988), 301-309. 
8. J. E. Goodman, R. Pollack, and R. Wenger, Geometric transversal theory. In J. Pach, ed., New Trends in Discrete and Computational Geometry, pp. 163-198. Algorithms and Combinatorics, 10. Springer-Verlag, Berlin, 1993.

9. J. E. Goodman, R. Pollack, and R. Wenger, Bounding the number of geometric permutations induced by k-transversals. J. Combin. Theory Ser. A 75 (1996), 187-197.

10. B. Grünbaum, On common transversals. Arch. Math. (Basel) 9 (1958), 465-469.

11. B. Grünbaum, Personal communication, 1998.

12. H. Hadwiger, Ungelöste Probleme, No. 7. Elem. Math. 10 (1955), 111.

13. H. Hadwiger, H. Debrunner, and V. Klee, Combinatorial Geometry in the Plane. Holt, Rinehart, and Winston, New York, 1964.

14. V. Klee, T. Lewis, and B. Von Hohenbalken, Appollonius revisited: supporting spheres for sundered systems. Discrete Comput. Geom. 18 (1997), 385-395.

15. T. Lewis, B. Von Hohenbalken, and V. Klee, Common supports as fixed points. Geom. Dedicata 60 (1996), 277-281.

16. H. Tverberg, Proof of Grünbaum's conjecture on common transversals for translates. Discrete Comput. Geom. 4 (1989), 191-203.

17. R. Wenger, Geometric permutations and connected components. Tech. Rep. TR-90-50, DIMACS, 1990.

18. R. Wenger, Helly-type theorems and geometric transversals. In J. E. Goodman and J. O'Rourke, eds., Handbook of Discrete and Computational Geometry, pp. 63-82. CRC Press, Boca Raton, FL, 1997.

19. R. Wenger, Progress in geometric transversal theory. In B. Chazelle, J. E. Goodman, and R. Pollack, eds., Advances in Discrete and Computational Geometry, pp. 375-393. American Mathematical Society, Providence, RI, 1998.

Received January 25, 1999, and in revised form July 7, 1999. Online publication May 3, 2000. 